\title{
Fatores associados à funcionalidade nas atividades instrumentais de vida diária em
}

\section{idosos brasileiros}

\author{
Factors associated with instrumental activities of daily living functional capacity in brazilian older
}

adults

Factores asociados a la funcionalidad en las actividades instrumentales de la vida diaria en

personas mayores brasileñas

Recebido: 22/03/2021 | Revisado: 31/03/2021 | Aceito: 05/04/2021 | Publicado: 15/04/2021

Felipe Douglas Silva Barbosa

ORCID: https://orcid.org/0000-0001-8583-903X

Universidade Federal de Sergipe, Brasil E-mail: felipedouglas@live.com

Camilla Louise de Melo

ORCID: https://orcid.org/0000-0001-5869-4197

Universidade Federal de Sergipe, Brasil

E-mail: camilla.lm@hotmail.com

Roberto Jerônimo dos Santos Silva

ORCID: https://orcid.org/0000-0002-4578-7666

Universidade Federal de Sergipe, Brasil

E-mail: rjeronimoss@gmail.com

\begin{abstract}
Resumo
Introdução: A redução da capacidade funcional nas Atividades Instrumentais de Vida Diária pode acompanhar o processo de envelhecimento, as quais permitem independência para participação na comunidade. A avaliação dessas atividades parece ser mais sensível a processos iniciais de alterações na funcionalidade de idosos. Objetivo: Analisar os fatores associados à funcionalidade nas Atividades Instrumentais de Vida Diária em idosos brasileiros. Metodologia: Foram utilizados os dados referentes à população idosa da Pesquisa Nacional de Saúde, 2013. Utilizouse como variável desfecho a "Capacidade funcional nas Atividades Instrumentais de Vida Diária" e dependentes as condições sociodemográficas, saúde e estilo de vida e, risco cardiovascular. Utilizou-se a regressão linear múltipla para amostras complexas para a análise estatística. Resultados: Foram incluídos no estudo 11177 participantes. Foi observada para ambos os sexos associação significativa entre a "Capacidade Funcional nas Atividades Instrumentais de Vida Diária" e as variáveis "Idade", "Situação conjugal", "Percepção de saúde", "Queda nos últimos 12 meses", "consumo de álcool" e "Nível de Atividade Física". Para o sexo feminino, apenas a "Pressão arterial sistólica". Conclusão: Estiveram associadas à funcionalidade nas Atividades Instrumentais de Vida Diária no idosos condições sociodemográficas, de saúde e estilo de vida e, relacionadas ao risco cardiovascular.
\end{abstract}

Palavras-chave: Idosos; Capacidade funcional; Pesquisa nacional de saúde; Fatores de risco.

\begin{abstract}
Introduction: Functional capacity decrease in instrumental activities of daily living, which allows the independence to participate in the community, can be related to the aging process. The evaluation of these activities seems to be more sensitive to initial processes in changes on the functional capacity of the older adults. Objective: To analyze factors associated with instrumental activities of daily living functioning in Brazilian older adults' population. Methods: Data from people over $\geq 60$ years old who participated in the National Health Survey 2013 were used. The outcome variable was "Functional capacity in instrumental activities of daily living". As independent variables, sociodemographic, health and lifestyle and cardiovascular risk were used. For statistical analysis, multiple linear regression for complex samples was used. Results: It was included 11.177 participants. A significant association for both genders was observed between "Functional Capacity in instrumental activities of daily living" and the variables "Age", "Marital status", "Health perception", "Falls in the last 12 months", "Alcohol consumption" and "Level of Physical Activity". "Systolic High blood pressure" was only for females. Conclusions: Several conditions were related to the functional capacity in the older population including sociodemographic, health and lifestyle, and cardiovascular risk.
\end{abstract}

Keywords: Older adults; Functional capacity; National health survey; Risk factors. 


\begin{abstract}
Resumen
Introducción: La reducción de la capacidad funcional en las Actividades Instrumentales de la Vida Diaria puede acompañar el proceso de envejecimiento, lo que permite la independencia para la participación en la comunidad. La evaluación de estas actividades parece ser más sensible a los procesos iniciales de cambios en la funcionalidad de las personas mayores. Objetivo: Analizar los factores asociados a la funcionalidad en las Actividades Instrumentales de la Vida Diaria en ancianos brasileños. Metodología: Se utilizaron datos de la población anciana de la Encuesta Nacional de Salud 2013. La variable de resultado fue "Capacidad funcional en actividades instrumentales de la vida diaria" y dependiente de las condiciones sociodemográficas, salud y estilo de vida y riesgo cardiovascular. Se utilizó regresión lineal múltiple para muestras complejas para análisis estadístico. Resultados: Se incluyeron 11.177 participantes en el estudio. Para ambos sexos se observó asociación significativa entre "Capacidad funcional en las actividades instrumentales de la vida diaria" y las variables "Edad", "Estado civil", "Percepción de salud", "Caída en los últimos 12 meses", "Consumo de alcohol "Y" Nivel de actividad física ". Para el sexo femenino, solo la "presión arterial sistólica". Conclusión: Se asociaron con funcionalidad en Actividades Instrumentales de la Vida Diaria en ancianos, condiciones sociodemográficas, de salud y estilo de vida, y relacionadas con riesgo cardiovascular.
\end{abstract}

Palabras clave: Ancianos; Capacidad funcional; Encuesta nacional en salud; Factores de riesgo.

\title{
1. Introdução
}

Grandes mudanças na população mundial têm ocorrido nos últimos anos, principalmente nos países em desenvolvimento, como no Brasil. O envelhecimento populacional, subsidiado pelas transições epidemiológica e demográfica, tem demonstrado os grandes avanços na assistência à população (Miranda et al., 2016; Organização Mundial da Saúde, 2015).

O envelhecimento não apresenta uma linearidade de condições de saúde e dependência em relação à idade cronológica, sendo possível a manutenção da capacidade funcional e boas condições de saúde da população idosa com a adoção de hábitos saudáveis durante a vida e condições ambientais favoráveis (Organização Mundial da Saúde, 2015). No entanto, quando esta relação não se apresenta de forma harmoniosa, pode estar associada ao desenvolvimento de doenças crônicas e eventos adversos de saúde, que poderão influenciar diretamente na capacidade funcional desta população (Sociedade Brasileira de Geriatria e Gerontologia, 2008).

A capacidade funcional pode ser definida como relação positiva entre as condições de saúde apresentadas pelo indivíduo e o ambiente ao qual está inserido, permitindo a participação e realização de atividades cotidianas (Classificação Internacional de Funcionalidade, Incapacidade e Saúde, 2008). A capacidade funcional dos idosos têm sido bastante estudada, tendo as avaliações das Atividades Instrumentais de Vida Diária (AIVDs) demonstrado resultados válidos e confiáveis (Alves et al., 2008b). As AIVDs podem ser definidas como "atividades de apoio à vida diária dentro de casa e na comunidade, que muitas vezes necessitam de interações mais complexas que as utilizadas nas Atividades de Vida diária (AVDs)", que são aquelas voltadas para o autocuidado ("Estrutura da prática da terapia ocupacional: domínio \& processo - 3ed," 2015). As AIVDs permitem ao indivíduo independência para participação na comunidade e estão relacionadas a tarefas como o gerenciamento da comunicação, financeiro e de saúde, estabelecimento e gerenciamento do lar, preparação de refeições, fazer compras, entre outras (“Estrutura da prática da terapia ocupacional: domínio \& processo - 3ed,” 2015).

Devido à complexidade das habilidades necessárias para a realização das AIVDs, a avaliação dessas atividades parece demonstrar grande sensibilidade a processos iniciais que culminam em alterações na capacidade funcional de idosos. Esta constatação pode ser demostrada pela maior prevalência de incapacidade funcional nas AIVDs na população idosa quando comparada com as atividades de vida diária (AVDs) (Cruz et al., 2016; Maciel \& Guerra, 2007; J. D. Nunes et al., 2017). Sabese que vários fatores tem sido associados à capacidade funcional do idosos nas AIVDs, incluindo variáveis sociodemográficas como a idade, sexo, grau de instrução, renda e estado civil, ocorrência de doenças crônicas, transtornos mentais e quedas, participação em atividades sociais, além da percepção de saúde e qualidade de vida (Fiedler \& Peres, 2008; Nunes et al., 2009; Pereira et al., 2011, 2012). No entanto, a grande maioria desses trabalhos apresentam amostras pequenas e limitadas, o que impede a generalização desses achados.

Mesmo os estudos que utilizaram amostras representativas da população brasileira, com utilização de grandes bancos 
populacionais, apresentam lacunas no que se refere às condições que podem estar associadas à manutenção da capacidade funcional nas AIVDs dos idosos, como em relação aos fatores relacionados ao estilo de vida (Alves et al., 2008a; Zanesco et al., 2018). Dentre esses fatores, a prática de atividade física tem demonstrado papel importante na manutenção ou melhora da capacidade funcional da população idosa (Filho et al., 2020; Presa et al., 2020; Siqueira et al., 2020). Wang e cols.(2019) relatam que quanto maior o tempo dispendido em atividade física, maior a capacidade funcional na realização das AIVDs, demonstrando uma maior independência na comunidade.

Com isso, faz-se necessária uma melhor compreensão dos fatores que estão associados à manutenção destas atividades. Assim, este trabalho tem como objetivo identificar os fatores associados à funcionalidade de idosos brasileiros nas AIVDs através da Pesquisa Nacional de Saúde, 2013.

\section{Metodologia}

O estudo foi realizado a partir de dados secundários que caracterizam a Pesquisa Nacional de Saúde - 2013, sendo esta de base domiciliar e desenvolvida em âmbito nacional pelo Ministério da Saúde em parceria com o Instituto Brasileiro de Geografia e Estatística (IBGE), no período entre agosto de 2013 e dezembro de 2014 (Damacena et al., 2015).

A amostra da PNS foi selecionada por conglomerados em três estágios, sendo primeiramente eleitas as Unidades Primárias de Amostragem, em seguida os domicílios e por fim foi selecionado um morador adulto. Todos os processos de seleção foram realizados de forma randômica. Artigos previamente publicados relataram a construção da PNS e de seu plano de amostragem (Damacena et al., 2015; Souza-Júnior et al., 2015). O questionário elaborado para realização da PNS foi subdividido em três partes: domiciliar, relativa a todos os moradores do domicílio e a parte individual. No total, foram realizadas 64348 entrevistas domiciliares e 60202 com o morador selecionado (Damacena et al., 2015).

Para este estudo, foram utilizados os dados referentes apenas à população idosa ( $\geq 60$ anos). A variável desfecho "Capacidade funcional nas AIVDs" foi obtida através da união de cinco questões do questionário da PNS, "Módulo K", seguindo o modelo estabelecido por Zanesco e cols (2018) realizando o somatório das pontuações das respostas. As perguntas estão apresentadas no Quadro 1. As questões utilizaram as seguintes opções de resposta: "1. Não consegue"," 2. Tem grande dificuldade", "3. Tem pequena dificuldade" e "4. Não tem dificuldade", sendo que a pergunta sobre "tomar os remédios sozinho(a)" incluiu a opção "5. Não faz uso de medicamentos".

Quadro 1. Perguntas utilizadas para obtenção da variável "Capacidade funcional nas AIVDs".

\begin{tabular}{|l|l|}
\hline \multirow{3}{*}{} & $\begin{array}{l}\text { "K22. Em geral, que grau de dificuldade tem para fazer compras sozinho(a), por exemplo de alimentos, roupas ou } \\
\text { medicamentos?" }\end{array}$ \\
\cline { 2 - 3 } & $\begin{array}{l}\text { "K25. Em geral, que grau de dificuldade tem para administrar as finanças sozinho(a) (cuidar do seu próprio } \\
\text { dinheiro)?" }\end{array}$ \\
\cline { 2 - 3 } & "K28. Em geral, que grau de dificuldade tem para tomar os remédios sozinho(a)?" \\
\cline { 2 - 3 } & "K.31. Em geral, que grau de dificuldade tem para ir ao médico sozinho(a)?" \\
\cline { 2 - 3 } & $\begin{array}{l}\text { "K34. Em geral, que grau de dificuldade tem para sair sozinho(a) utilizando um transporte como ônibus, metrô, táxi, } \\
\text { carro, etc.?" }\end{array}$ \\
\end{tabular}

Fonte: Autores.

Os dados da "Capacidade funcional nas AIVDs" foram utilizados de forma contínua, sendo considerados os idosos com "melhor capacidade funcional" os que apresentaram maior pontuação na união das questões e com "pior capacidade funcional", os que apresentaram menor pontuação. No que concerne às variáveis independentes, foram dicotomizadas de acordo com o Quadro 2. 
Quadro 2. Quadro de dicotomização das variáveis categóricas.

\begin{tabular}{|c|c|c|}
\hline VARIÁVEIS & $\begin{array}{l}\text { DICOTOMIZAÇÃO } \\
\text { UTILIZADA }\end{array}$ & REFERÊNCIA PARA DICOTOMIZAÇÃO \\
\hline \multirow{2}{*}{ Idade } & Idosos jovens & \multirow{2}{*}{$\begin{array}{c}\text { A variável foi retirada da pergunta "C8. Idade".a As respostas foram dicotomizadas em: } \\
\text { Idosos jovens: } \leq 70 \text { anos } \\
\text { Idosos velhos: }>70 \text { anos }\end{array}$} \\
\hline & Idosos velhos & \\
\hline \multirow{2}{*}{ Situação conjugal } & $\begin{array}{c}\text { Tem relacionamento } \\
\text { oficializado }\end{array}$ & \multirow{2}{*}{$\begin{array}{l}\text { A variável foi retirada da pergunta "C11. Qual o estado civil de_?"a. A resposta } 1 \\
\text { (casado) foi transformada em "Tem relacionamento oficializado" e as entre } 2 \text { e } 5 \\
\text { (separado, divorciado, viúvo, solteiro, respectivamente) foram categorizados como } \\
\text { "Não tem relacionamento oficializado". }\end{array}$} \\
\hline & $\begin{array}{l}\text { Não tem relacionamento } \\
\text { oficializado }\end{array}$ & \\
\hline \multirow{2}{*}{ Raça } & Brancos & \multirow{2}{*}{$\begin{array}{l}\text { A variável foi retirada da pergunta "C9. Cor ou raça"a. A resposta } 1 \text { permaneceu } \\
\text { "branco" e as respostas entre } 2 \text { e } 5 \text { (preta, amarela, parda e indígena, respectivamente) } \\
\text { foram categorizadas como "não brancos". }\end{array}$} \\
\hline & Não brancos & \\
\hline \multirow{2}{*}{$\begin{array}{l}\text { Percepção de estado } \\
\text { de saúde }\end{array}$} & Boa & \multirow{2}{*}{$\begin{array}{l}\text { A variável foi retirada da pergunta "N1. Em geral, como o(a) sr(a) avalia a sua } \\
\text { saúde?"a. As respostas } 1 \text { e } 2 \text { foram categorizadas como percepção "boa" e as respostas } \\
\text { entre } 3 \text { e } 5 \text { percepção "ruim". }\end{array}$} \\
\hline & Ruim & \\
\hline \multirow{2}{*}{$\begin{array}{l}\text { Ocorrência de } \\
\text { quedas nos últimos } \\
12 \text { meses }\end{array}$} & Sim & \multirow[t]{2}{*}{$\begin{array}{c}\text { A variável foi retirada da pergunta "K54. Nos últimos } 12 \text { meses, } \\
\text { queda que o(a) levou a procurar o serviço de saúde?" A resposta } 1 \text { permaneceu "Sim" e } \\
\text { a resposta } 2 \text { permaneceu "Não". }\end{array}$} \\
\hline & Não & \\
\hline \multirow[t]{2}{*}{ Tempo de tela } & Até 2 horas por dia & \multirow[t]{2}{*}{$\begin{array}{l}\text { A variável foi retirada da pergunta "P45. Em média, quantas horas por dia o(a) sr(a) } \\
\text { costuma ficar assistindo televisão?"a. As respostas } 1 \text {, } 2 \text { e } 8 \text {, foram categorizadas como } \\
\text { "Até } 2 \text { horas por dia" tempo de tela ( } \leq 2 \text { horas por dia) e as respostas entre } 3 \text { e } 7 \text { "Mais } \\
\text { que } 2 \text { horas por dia" tempo de tela ( }>2 \text { horas por dia). }\end{array}$} \\
\hline & Mais que 2 horas por dia & \\
\hline \multirow{2}{*}{ Consumo de álcool } & Sim & \multirow{2}{*}{$\begin{array}{l}\text { A variável foi retirada da pergunta "P27. Com que frequência o(a) sr(a) costuma } \\
\text { consumir alguma bebida alcoólica?"a. As respostas } 1 \text { permaneceu como "Não" e as } \\
\text { respostas } 2 \text { e 3, foram categorizadas como "Sim" }\end{array}$} \\
\hline & Não & \\
\hline \multirow[t]{2}{*}{ Consumo de tabaco } & Sim & \multirow{2}{*}{$\begin{array}{l}\text { A variável foi retirada da pergunta "P50. Atualmente, o(a) sr(a) fuma algum produto do } \\
\text { tabaco?"a. As respostas } 1 \text { e } 2 \text {, foram categorizadas como "Sim" e a resposta } 3 \\
\text { permaneceu como "Não". }\end{array}$} \\
\hline & Não & \\
\hline \multirow{2}{*}{ Atividade física } & Sedentário & \multirow{2}{*}{$\begin{array}{l}\text { A variável foi obtida através do somatório dos tempos dispendidos em atividade física } \\
\text { em todos os domínios (Lazer, deslocamento, doméstica e trabalho). O somatório foi } \\
\text { dicotomizado em: } \\
\text { Sedentário: }<150 \mathrm{~min} \\
\text { Ativo: } \geq 150 \mathrm{~min}\end{array}$} \\
\hline & Ativo & \\
\hline \multirow{2}{*}{$\begin{array}{l}\text { Circunferência de } \\
\text { cintura }\end{array}$} & Normal & \multirow{2}{*}{$\begin{array}{c}\text { A variável foi retirada da pergunta "W00301 - Informe a sua circunferência da cintura } \\
\text { (primeira medida em cm)".a As respostas foram dicotomizadas em: } \\
\text { Homens - Normal: } \leq 94 \text { centímetros } \\
\text { Homens - Aumentada: }>94 \text { centímetros } \\
\text { Mulheres - Normal: } \leq 80 \text { centímetros } \\
\text { Mulheres - Aumentada: }>80 \text { centímetros }\end{array}$} \\
\hline & Aumentada & \\
\hline \multirow[t]{2}{*}{$\begin{array}{l}\text { Pressão Arterial } \\
\text { sistólica }\end{array}$} & Normotenso & \multirow{2}{*}{$\begin{array}{l}\text { A variável foi retirada a partir das médias aritméticas das aferições realizadas. As } \\
\text { respostas foram dicotomizadas em: } \\
\text { Normotenso: } \leq 129 \mathrm{mmHg} \\
\text { Hipertenso: }>129 \mathrm{mmHg}\end{array}$} \\
\hline & Hipertenso & \\
\hline \multirow{2}{*}{$\begin{array}{l}\text { Pressão arterial } \\
\text { diastólica }\end{array}$} & Normotenso & \multirow{2}{*}{$\begin{array}{l}\text { A variável foi retirada a partir das médias aritméticas das aferições realizadas. As } \\
\text { respostas foram dicotomizadas em: } \\
\text { Normotenso: } \leq 80 \mathrm{mmHg} \\
\text { Hipertenso: }>80 \mathrm{mmHg}\end{array}$} \\
\hline & Hipertenso & \\
\hline
\end{tabular}

a : As questões dos estudo são apresentadas em escala Lickert. Fonte: Autores.

$\mathrm{Na}$ análise estatística, os dados da amostra foram estratificados de acordo com o sexo. A estatística descritiva foi realizada através de frequência simples e percentual. Foi utilizado o modelo estatístico de regressão linear múltipla para amostras complexas com objetivo de analisar os fatores associados à capacidade funcional nas AIVDs em idosos. No ajuste de calibração, foram incorporados os estratos, as unidades primárias de amostragem e os pesos amostrais conforme indicado por 
Souza-Junior e cols.13 A análise foi realizada no pacote estatístico Statistical Package for the Social Sciences (SPSS) versão 22, considerando um nível de significância p $<0,05$ e intervalo de confiança (IC) de $95 \%$.

O projeto da Pesquisa Nacional de Saúde foi aprovado pela Comissão Nacional de Ética em Pesquisa no dia 8 de julho de 2013, sob o n ${ }^{\circ}$ 10853812.7.0000.0008. Além disso, está de acordo com o estabelecido pela resolução do Conselho Nacional de Saúde n 466, de 12 de dezembro de 2012.

\section{Resultados}

A amostra desse estudo foi composta por 6.622 mulheres e 4.555 homens, totalizando 11.177 idosos. As características da amostra estão descritas na Tabela 1. Com relação às variáveis sociodemográficas, a maioria do sexo masculino é composta por idosos jovens $(61,2 \%)$, não brancos $(54,4 \%)$ e com relacionamento oficializado (59,4\%). Para o sexo feminino, a maioria é idosa jovem (59,8\%), não branca (51,1\%) e não tem relacionamento oficializado (68,2\%).

Para as variáveis relacionadas à saúde e estilo de vida, a maioria dos idosos do sexo masculino apresenta uma percepção ruim da saúde (54\%), não sofreu quedas nos últimos 12 meses (94,1\%), com tempo de tela menor que 2 horas por dia $(52,8 \%)$, consumidora de álcool $(77,1 \%)$, não consumidora de tabaco $(82,8 \%)$ e sedentária $(59,3 \%)$. A maioria das idosas tem uma percepção ruim da saúde (57,5\%), não sofreu quedas nos últimos 12 meses $(90,3 \%)$, com tempo de tela maior que 2 horas por dia (58,3\%), consumidora de álcool (93,9\%), não consumidora de tabaco $(90,6 \%)$ e sedentária $(62,1 \%)$.

Por fim, para as variáveis de marcadores de risco cardiovascular, os idosos do sexo masculino apresentaram "Circunferência de cintura" categorizada como normal (58,4\%), normotensos para "Pressão arterial sistólica" (65,5\%) e hipertensos para "Pressão arterial diastólica" $(81,6 \%)$. Para o sexo feminino, a maioria tinha circunferência de cintura elevada (86,1\%), normotensas para "Pressão arterial sistólica" (64,9\%) e hipertensas para "Pressão arterial diastólica" (81,8\%). 
Tabela 1. Características da população do estudo (N: 11.777).

\begin{tabular}{|c|c|c|c|c|}
\hline Variáveis & $\begin{array}{c}\text { Homens } \\
\mathbf{N}(\%)\end{array}$ & IC $(95 \%)$ & $\begin{array}{l}\text { Mulheres } \\
\mathbf{N}(\%)\end{array}$ & IC (95\%) \\
\hline $\begin{array}{l}\text { Idade } \\
\text { Idosos jovens } \\
\text { Idosos velhos }\end{array}$ & $\begin{array}{l}2788(61,2) \\
1767(38,8)\end{array}$ & $(37,38-40,21)$ & $\begin{array}{l}3900(59,8) \\
2722(40,1)\end{array}$ & $(39,92-42,29)$ \\
\hline $\begin{array}{l}\text { Raça } \\
\text { Brancos } \\
\text { Não brancos }\end{array}$ & $\begin{array}{l}2075(45,6) \\
2480(54,4)\end{array}$ & $(53,00-55,89)$ & $\begin{array}{l}3239(48,9) \\
3383(51,1)\end{array}$ & $(49,88-52,29)$ \\
\hline $\begin{array}{l}\text { Situação conjugal } \\
\text { Tem relacionamento oficializado } \\
\text { Não tem relacionamento oficializado }\end{array}$ & $\begin{array}{l}2705(59,4) \\
1850(40,6)\end{array}$ & $(39,19-42,04)$ & $\begin{array}{l}2103(31,8) \\
4519(68,2)\end{array}$ & $(67,12-69,36)$ \\
\hline $\begin{array}{l}\text { Percepção do estado de saúde } \\
\text { Boa } \\
\text { Ruim }\end{array}$ & $\begin{array}{l}2094(46,0) \\
2461(54,0)\end{array}$ & $(52,58-55,48)$ & $\begin{array}{l}2813(42,5) \\
3809(57,5)\end{array}$ & $(56,33-58,71)$ \\
\hline $\begin{array}{l}\text { Queda nos últimos } 12 \text { meses } \\
\text { Sim } \\
\text { Não }\end{array}$ & $\begin{array}{l}270(05,9) \\
4285(94,1)\end{array}$ & $(93,00-95,00)$ & $\begin{array}{l}641(09,7) \\
5981(90,3)\end{array}$ & $(90,00-91,00)$ \\
\hline $\begin{array}{l}\text { Tempo de tela } \\
\text { Até } 2 \text { horas por dia } \\
\text { Mais que } 2 \text { horas por dia }\end{array}$ & $\begin{array}{l}2406(52,8) \\
2149(47,2)\end{array}$ & $(45,73-48,63)$ & $\begin{array}{l}2759(41,7) \\
3863(58,3)\end{array}$ & $(57,15-59,52)$ \\
\hline $\begin{array}{l}\text { Consumo de álcool } \\
\text { Sim } \\
\text { Não }\end{array}$ & $\begin{array}{l}3510(77,1) \\
1045(22,9)\end{array}$ & $(21,72-24,16)$ & $\begin{array}{l}6218(93,9) \\
404(06,1)\end{array}$ & $(05,52-06,68)$ \\
\hline $\begin{array}{l}\text { Consumo de tabaco } \\
\text { Sim } \\
\text { Não }\end{array}$ & $\begin{array}{l}784(17,2) \\
3771(82,8)\end{array}$ & $(81,69-83,88)$ & $\begin{array}{l}620(09,4) \\
6002(90,6)\end{array}$ & $(89,94-91,34)$ \\
\hline $\begin{array}{l}\text { Nível de AF } \\
\text { Sedentário } \\
\text { Ativo }\end{array}$ & $\begin{array}{l}2702(59,3) \\
1853(40,7)\end{array}$ & $(39,25-42,11)$ & $\begin{array}{l}4115(62,1) \\
2507(37,1)\end{array}$ & $(36,69-39,03)$ \\
\hline $\begin{array}{l}\text { Circunferência de cintura* } \\
\text { Normal } \\
\text { Aumentada }\end{array}$ & $\begin{array}{l}2246(58,4) \\
1061(41,6)\end{array}$ & $(40,06-43,18)$ & $\begin{array}{l}775(13,9) \\
4815(86,1)\end{array}$ & $(85,23-87,04)$ \\
\hline $\begin{array}{l}\text { Pressão arterial sistólica* } \\
\text { Normotenso } \\
\text { Hipertenso }\end{array}$ & $\begin{array}{l}2536(65,5) \\
1337(34,5)\end{array}$ & $(33,02-36,02)$ & $\begin{array}{l}3616(64,9) \\
1957(35,1)\end{array}$ & $(33,86-36,37)$ \\
\hline $\begin{array}{l}\text { Pressão arterial diastólica* } \\
\text { Normotenso } \\
\text { Hipertenso }\end{array}$ & $\begin{array}{l}709(18,4) \\
3141(81,6)\end{array}$ & $(80,36-82,81)$ & $\begin{array}{l}1009(18,2) \\
4529(81,8)\end{array}$ & $(80,76-82,80)$ \\
\hline
\end{tabular}

*Não foram coletadas as informações de todos os participantes para estas variáveis. Fonte: Autores.

Na Tabela 2 estão apresentados os resultados obtidos na regressão linear múltipla para o sexo masculino, onde observa-se associação significativa entre a "Capacidade Funcional nas AIVDs" e as variáveis "Idade” ( $\mathrm{p} \leq 0,001)$, "Situação conjugal” ( $\mathrm{p}=0,005)$, "Percepção de saúde” $(\mathrm{p} \leq 0,001)$, “Queda nos últimos 12 meses” ( $\mathrm{p}=0,010)$, “Consumo de álcool” ( $\mathrm{p} \leq$ 0,001) e "Nível de AF" ( $\mathrm{p} \leq 0,001)$. 
Tabela 2. Resultado da regressão linear múltipla com relação aos fatores associados à funcionalidade nas AIVDs em homens idosos brasileiros.

\begin{tabular}{|c|c|c|c|c|c|}
\hline \multirow[t]{2}{*}{ Variáveis } & \multirow[t]{2}{*}{$\mathbf{B}$} & \multirow[t]{2}{*}{$\begin{array}{c}\text { Erro } \\
\text { Padrão }\end{array}$} & \multicolumn{2}{|c|}{$\begin{array}{c}\text { Intervalo de confiança } \\
(95 \%)\end{array}$} & \multirow[t]{2}{*}{$\mathbf{p}$} \\
\hline & & & Mínimo & Máximo & \\
\hline (Constante) & 18.806 & 0.579 & 17.670 & 19.941 & $\leq 0.001$ \\
\hline Idade & -1.201 & 0.162 & -1.519 & -0.883 & $\leq \mathbf{0 . 0 0 1}$ \\
\hline Raça & 0.098 & 0.152 & -0.201 & 0.397 & .521 \\
\hline Situação conjugal & -0.452 & 0.160 & -0.766 & -0.138 & 0.005 \\
\hline Percepção de saúde & -1.272 & 0.138 & -1.543 & -1.001 & $\leq \mathbf{0 . 0 0 1}$ \\
\hline $\begin{array}{l}\text { Ocorrência de quedas nos } \\
\text { últimos } 12 \text { meses }\end{array}$ & 1.352 & 0.521 & 0.330 & 2.375 & 0.010 \\
\hline Tempo de tela & 0.069 & 0.147 & -0.220 & 0.358 & 0.640 \\
\hline Consumo de álcool & 0.483 & 0.116 & 0.256 & 0.711 & $\leq \mathbf{0 . 0 0 1}$ \\
\hline Consumo de tabaco & -0.189 & 0.180 & -0.542 & 0.165 & 0.295 \\
\hline Nível de atividade física & 0.968 & 0.116 & 0.741 & 1.194 & $\leq \mathbf{0 . 0 0 1}$ \\
\hline Circunferência de cintura & -0.299 & 0.164 & -0.621 & 0.023 & 0.068 \\
\hline Pressão arterial sistólica & -0.076 & 0.175 & -0.420 & 0.267 & 0.663 \\
\hline Pressão arterial diastólica & 0.126 & 0.162 & -0.191 & 0.444 & 0.435 \\
\hline
\end{tabular}

Fonte: Autores.

Na Tabela 3 estão apresentados os resultados obtidos na regressão linear múltipla para o sexo feminino, onde observase associação significativa entre a "Capacidade Funcional nas AIVDs" e as variáveis "Idade” (p $\leq$ 0,001), "Situação conjugal” ( $\mathrm{p} \leq 0,001)$, "Percepção de saúde" ( $\mathrm{p} \leq 0,001)$, “Queda nos últimos 12 meses" ( $\mathrm{p} \leq 0,001)$, “Consumo de álcool” ( $\mathrm{p}=0,018)$, "Nível de AF" ( $\mathrm{p} \leq 0,001)$ e "Pressão arterial sistólica" $(\mathrm{p}=0,015)$.

Tabela 3. Resultado da regressão linear múltipla com relação aos fatores associados à funcionalidade nas AIVDs em mulheres idosas brasileiras.

\begin{tabular}{cccccc}
\hline Variáveis & \multirow{2}{*}{ B } & $\begin{array}{c}\text { Erro } \\
\text { Padrão }\end{array}$ & \multicolumn{2}{c}{$\begin{array}{c}\text { Intervalo de confiança } \\
(\mathbf{9 5 \%})\end{array}$} & p \\
\hline (Constante) & & & Mínimo & Máximo & \\
Idade & 18.337 & 0.506 & 17.345 & 19.329 & $\leq \mathbf{0 . 0 0 1}$ \\
Raça & -1.952 & 0.184 & -2.312 & -1.592 & $\leq \mathbf{0 . 0 0 1}$ \\
Situação conjugal & -0.069 & 0.172 & -0.406 & 0.267 & 0.687 \\
Percepção de saúde & -0.721 & 0.160 & -1.035 & -0.407 & $\leq \mathbf{0 . 0 0 1}$ \\
Ocorrência de quedas nos & -1.352 & 0.155 & -1.656 & -1.048 & $\leq \mathbf{0 . 0 0 1}$ \\
últimos 12 meses & 1.259 & 0.348 & 0.577 & 1.940 & $\leq \mathbf{0 . 0 0 1}$ \\
Tempo de tela & 0.321 & 0.173 & -0.017 & 0.659 & 0.063 \\
Consumo de álcool & 0.464 & 0.196 & 0.079 & 0.849 & $\mathbf{0 . 0 1 8}$ \\
Consumo de tabaco & 0.024 & 0.279 & -0.523 & 0.570 & 0.933 \\
Nível de atividade física & 1.564 & 0.146 & 1.277 & 1.851 & $\leq \mathbf{0 . 0 0 1}$ \\
Circunferência de cintura & -0.239 & 0.245 & -0.719 & 0.242 & 0.330 \\
Pressão arterial sistólica & 0.424 & 0.174 & 0.082 & 0.766 & $\mathbf{0 . 0 1 5}$ \\
Pressão arterial diastólica & 0.117 & 0.246 & -0.365 & 0.600 & 0.634 \\
& & & & & \\
\hline
\end{tabular}

Fonte: Autores.

\section{Discussão}

Este estudo teve como objetivo principal analisar os fatores associados à capacidade funcional em idosos brasileiros nas AIVDs. Conforme citado anteriormente, a partir dos resultados obtidos na PNS, verificou-se que quanto maior a pontuação na variável "Capacidade Funcional" nas AIVDs, melhor a capacidade funcional do idoso. 
Em relação às condições sociodemográficas, as variáveis "Idade", "Sexo" e "Situação conjugal" apresentaram associação significativa com a variável desfecho. Para a variável "Idade", os resultados demonstraram que idosos mais novos apresentaram melhor capacidade funcional nas AIVDs. Este resultado é bastante encontrado em outros estudos (Cruz et al., 2016; Maciel \& Guerra, 2007; Nunes et al., 2017; Santos et al., 2014) e demostra o efeito do declínio das capacidades intrínsecas com a longevidade na capacidade funcional do idoso (Organização Mundial da Saúde, 2015). Considerando que os processos de transição demográfica e epidemiológica continuarão ocorrendo, haverá um aumento no número de idosos longevos no país, sendo assim necessárias intervenções que visem prevenir os efeitos deletérios que podem acompanhar o envelhecimento da população.

Para a variável "Situação conjugal", ter relacionamento oficializado esteve associado a melhor funcionalidade nas AIVDs. Outras pesquisas reforçam estes achados, já que relatam que solteiros (Maciel \& Guerra, 2007) e viúvos (M. C. R. Nunes et al., 2009; Pereira et al., 2011) apresentam piores níveis de capacidade funcional nas AIVDs, quando comparados a pessoas casadas.

Para as variáveis relacionadas à condição de saúde e estilo de vida, foram associadas a capacidade funcional nas AIVDs à "Percepção de saúde", "Quedas nos últimos 12 meses", "Consumo de álcool" e "Nível de AF". No que concerne a percepção de saúde, ter melhor percepção de saúde esteve associado a melhor funcionalidade nas AIVDs. Este resultado foi encontrado por outros autores (Maciel \& Guerra, 2007; Rosa et al., 2003; Silva et al., 2012) e reforça a concepção de que, para o idoso, a funcionalidade seja o componente que tem maior relevância na percepção de seu estado de saúde.

Com relação à ocorrência de quedas, nesse estudo, a melhor capacidade funcional esteve associada a não ocorrência de quedas nos últimos 12 meses. Além disso, para os idosos do sexo masculino esta condição apresentou o maior resultado de “ $\beta$ ” na análise estatística, demonstrando a influência desta variável para a melhor capacidade funcional nas AIVDs. As quedas podem ocorrer como consequência da incapacidade funcional nas AIVDs, ou seja, existe um risco maior de quedas devido a incapacidade funcional, bem como sua ocorrência pode ser responsável por essa incapacidade (Gomes, 2018; Santos et al., 2014). Além disso, devido as consequências, tanto físicas, como mentais e sociais, as quedas estão relacionadas a maior risco de hospitalização, morbidade e mortalidade (Maia et al., 2011).

O consumo de álcool também esteve associado à melhor funcionalidade nas AIVDs, cuja explicação deriva da necessidade de boa capacidade funcional para que o idoso possa ter acesso à bebida alcoólica e realizar o seu consumo, principalmente em atividades como a mobilidade na comunidade, para, por exemplo, fazer a compra da bebida alcoólica em algum estabelecimento comercial. No entanto, não foram analisados, neste estudo, o tempo e a quantidade consumida por esses idosos. Este fator pode ter influenciado no resultado apresentado, já que vários autores relatam os efeitos nocivos que o consumo de álcool pode apresentar na saúde dos idosos, com influência direta na sua funcionalidade e qualidade de vida (Andrade \& Oliveira, 2009; Domingues \& Lopes, 2018; Sequeto et al., 2018; Soares et al., 2016).

Com relação à $\mathrm{AF}$, o maior tempo de prática em todos os domínios esteve associado à maior capacidade funcional nas AIVDs em idosos brasileiros. Além disso, ser ativo fisicamente apresentou o maior valor de " $\beta$ " na regressão linear para o sexo feminino, demonstrando que esta condição apresenta maior influência para melhor capacidade funcional nas AIVDs nas idosas brasileiras.

De forma geral, a AF apresenta inúmeros benefícios à saúde da população idosa. Vários levantamentos demonstram que sua prática influencia na manutenção da capacidade funcional nas AIVDs (de Queiroz et al., 2014; Pereira et al., 2011; Virtuoso et al., 2012). Esses estudos quantificaram o nível de atividade física, buscando identificar a frequência e o tempo dedicados a esta prática em todos os seus domínios, concluindo que este é um preditor de capacidade funcional do idoso. O estudo de Virtuoso-Júnior e cols.(2012) sugeriu os pontos de corte de 280 minutos/semana para mulheres e 410 minutos/semana para predição de ausência de incapacidade funcional nos idosos. Sendo assim, esses estudos concluíram que 
ser ativo fisicamente está associado à melhor capacidade funcional nas AIVDs, corroborando com os resultados encontrados (de Queiroz et al., 2014; Pereira et al., 2011; Virtuoso et al., 2012).

Por fim, das variáveis relacionadas ao risco cardiovascular, apenas a variável "Pressão arterial sistólica" apresentou associação com a capacidade funcional para o sexo feminino, sugerindo que mulheres idosas hipertensas apresentam maior capacidade funcional nas AIVDs. Para esta pesquisa utilizou-se ponto corte para hipertensão arterial de $\geq 130 \mathrm{mmHg}$, conforme indica a Organização Pan-americana (Organização Pan-americana de Saúde, 2018) de Saúde e a American Heart Association (American Heart Association, 2017) atualmente, porém quando esta pesquisa foi realizada o ponto de corte utilizado para definição de hipertensão arterial era maior $(\geq 140 \mathrm{mmHg})$, fato que pode ter influenciado nos resultados.

Como limitação, ressalta-se que nosso estudo incluiu os idosos solteiros e viúvos na categoria "Não tem relacionamento oficializado" na dicotomização da variável "Situação conjugal", o que pode ter influenciado nos resultados encontrados para esta variável. Porém, outros estudos com a mesma população e com metodologias diferentes, também identificaram que viver com cônjuge está associado a melhor capacidade funcional nas AIVDs, situação que valida os resultados encontrados (Rocha et al., 2017; Zanesco et al., 2018).

\section{Considerações Finais}

Levando em conta os aspectos analisados, esse estudo constatou que grande variedade de fatores está associada à funcionalidade nas AIVDs de idosos brasileiros, incluindo os fatores sociodemográficas, os relacionados à saúde e estilo de vida, bem como ao risco cardiovascular. Este trabalho ressalta a importância que os fatores modificáveis apresentaram, pois são capazes de influenciar na manutenção da capacidade funcional do idoso nas AIVDs na população brasileira, tais como a prática de AF em todos os domínios, que apresenta relação direta com a capacidade funcional nas AIVDs.

Além disso, sugere-se que pesquisas, principalmente ensaios clínicos randomizados, sejam realizadas a fim de comprovar que intervenções voltadas para as condições modificáveis são efetivas na manutenção na capacidade funcional do idoso para realização de suas AIVDs. Assim, estas poderão subsidiar o planejamento e a implantação de políticas públicas que busquem a melhora da qualidade de vida da população idosa no Brasil.

\section{Referências}

Alves, L. C., Leite, I. C., \& Machado, C. J. (2008a). Perfis de saúde dos idosos no Brasil: análise da Pesquisa Nacional por Amostra de Domicílios de 2003 utilizando o método Grade of Membership. Cad. Saúde Pública, 24(3), 535-546.

Alves, L. C., Leite, I. C., \& Machado, C. J. (2008b). The concept and measurement of functional disability in the elderly population : a literature review. Ciência \& Saúde Coletiva, 13(4), 1199-1207.

American Heart Association. (2017). Understanding Blood Pressure Readings. https://www.heart.org/en/health-topics/high-blood-pressure/understandingblood-pressure-readings

Andrade, A. G. de, \& Oliveira, L. G. de. (2009). Principais conseqüências em longo prazo relacionadas ao consumo moderado de álcool. In J. C. Anthony, A. G. de Andrade, \& C. M. Silveira (Eds.), Álcool e suas consequências: uma abordagem multiconceitual. Minha Editora.

Classificação Internacional de Funcionalidade, Incapacidade e Saúde. (2008). Editora da Universidade de São Paulo.

Cruz, D. T., Leite, I. G., Barbosa, M. B., \& Leite, I. C. G. (2016). Disability prevalence functional and sociodemographic factors associated in out of judge of elderly, MG. Revista Kairós Gerontologia, 19(22), 9-28.

Damacena, G. N., Szwarcwald, C. L., Malta, D. C., Júnior, P. R. B. de S., Vieira, M. L. F. P., Pereira, C. A., Neto, O. L. de M., \& Júnior, J. B. S. (2015). O processo de desenvolvimento da Pesquisa Nacional de Saúde no Brasil, 2013. Epidemiol. Serv. Saude, 24(2), 197-206. https://doi.org/10.5123/S167949742015000200002

de Queiroz, B. M., da Coqueiro, R. S., Leal Neto, J. de S., Borgatto, A. F., Barbosa, A. R., \& Fernandes, M. H. (2014). Inatividade física em idosos não institucionalizados: Estudo de base populacional. Ciencia e Saude Coletiva, 19(8), 3489-3496. https://doi.org/10.1590/1413-81232014198.19882013

Domingues, M. P. S., \& Lopes, J. do C. M. (2018). Álcool: o uso abusivo entre idosos e comprometimento na qualidade de vida. Revista Gestao \& Saude, 19(1), 69-88. 
Estrutura da prática da terapia ocupacional: domínio \& processo - 3ed. (2015). In Rev Ter Ocup Univ São Paulo (Vol. 26). https://doi.org/http://dx.doi.org/10.11606/issn.2238-6149.v26iespp1-49

Fiedler, M. M., \& Peres, K. G. (2008). Functional status and associated factors among the elderly in a southern Brazilian city : a population- based study. Cad. Saúde Pública, 24(2), 409-415.

Filho, B. F. de L., Perracini, M. R., Formiga, B. B. S., Sousa, A. G. P. de, Cavalcanti, F. A. da C., \& Gazzola, J. M. (2020). Carga de fragilidade em idosos com Diabetes Mellitus tipo 2 e fatores relacionados Frailty burden in older adults with type 2 Diabetes Mellitus and related factors Carga de fragilidad en personas mayores con Diabetes Mellitus tipo 2 y factores relacionado. Research, Society and Development, 9(8), 1-20. https://doi.org/10.33448/rsd-v9i8.5645

Gomes, M. de L. de F. (2018). Atividades instrumentais de vida diária e risco de quedas em pessoas idosas participantes do centro de convivência do idoso no município de Vitória da Conquista-BA. Escola Bahiana de Medicina e Saúde Pública.

Maciel, Á. C. C., \& Guerra, R. O. (2007). Influência dos fatores biopsicossociais sobre a capacidade funcional de idosos residentes no nordestes do Brasil. Rev Bras Epidemiol, 10(2), 178-189.

Maia, B. C., Viana, P. S., Maria, P., Arantes, M., \& Alencar, M. A. (2011). Consequências das Quedas em Idosos Vivendo na Comunidade. Rev. Bras. Geriatr. Gerontol, 14(2), 381-393.

Miranda, G. M. D., Mendes, A. C. G., \& Silva, A. L. A. da. (2016). O envelhecimento populacional brasileiro: desafios e consequências sociais atuais e futuras. Revista Brasileira de Geriatria e Gerontologia, 19(3), 507-519. https://doi.org/10.1590/1809-98232016019.150140

Nunes, J. D., Saes, M. de O., Nunes, B. P., Siqueira, F. C. V., Soares, D. C., Fassa, M. E. G., Thumé, E., Facchini, L. A., \& 1Universidade. (2017). Functional disability indicators and associated factors in the elderly: a population-based study in Bagé, Rio Grande do Sul, Brazil. Epidemiol. Serv. Saude, 26(2), 295304. https://doi.org/10.5123/S1679-49742017000200007

Nunes, M. C. R., Ribeiro, R. C. L., Rosado, L. E. F. P. L., \& Franceschini, S. C. (2009). Influência das características sociodemográficas e epidemiológicas na capacidade funcional de idosos residentes em Ubá, Minas Gerais. Rev Bras Fisioter, São Carlos, 13(5).

Organização Mundial da Saúde. (2015). Relatório Mundial de Envelhecimento e Saúde. Organização Mundial de Saúde, 1, 1-29. https://doi.org/10.1017/CBO9781107415324.004

Organização Pan-americana de Saúde. (2018). O que é Hipertensão Arterial, Causas, Sintomas e Tratamento. https://opas.org.br/o-que-e-hipertensao-arterialcausas-sintomas-e-tratamento/

Pereira, G. N., Bastos, G. A. N., Del Duca, G. F., Bós, A. J. G., \& Bós, Â. J. G. (2012). Socioeconomic and demographic indicators associated with functional disability in the elderly. Cad Saude Publica, 28(11), 2035-2042. https://doi.org/10.1590/S0102-311X2012001100003

Pereira, G. N., Duca, G. F. Del, \& Bastos, G. A. N. (2011). Indicadores de saúde associados à incapacidade funcional em idosos de baixa renda. Geriatrics, Gerontology and Aging, 5(2), 66-73.

Presa, A. A., Alvarez, B. R., Mazon, J., \& Daminelli, S. A. (2020). Avaliação da capacidade funcional de idosas com artralgia praticantes de hidroginástica e cadastradas no programa de atenção multidisciplinar à saúde do idoso. Research, Society and Development, 9(10), 1-18. https://doi.org/10.33448/rsdv9i10.8972

Rocha, J. de P., Oliveira, G. G., Neris, J. C. D., Bós, A. M. G., \& Bós, Â. J. G. (2017). Impacto clínico, socioeconômico e da autopercepção de saúde na funcionalidade de idosos. Geriatrics, Gerontology and Aging, 11(3), 124-132. https://doi.org/10.5327/z2447-211520171700051

Rosa, T. E. da C., Benício, M. H. D., Latorre, M. do R. D. de O., \& Ramos, L. R. (2003). Determinant factors of functional status among the elderly. Rev Saúde Pública, 37(1), 40-48.

Santos, A. M. dos, Franco, S., \& Reis, M. A. M. (2014). Fatores associados à perda da capacidade funcional em idosos em município no sul do país. Geriatr Gerontol Aging, 8(1), 19-26.

Sequeto, G. S., Santos, N. A., Atalaia-silva, L., \& Mármora, C. H. C. (2018). Associação entre consumo de álcool , quedas e internação em idosos Artigo original. Revista Hospital Universitário Pedro Ernesto, 17(2), 45-52. https://doi.org/10.12957/rhupe.2018.40810

Silva, R. J. dos S., Smith-Menezes, A., Tribess, S., Rómo-Perez, V., \& Júnior, J. S. V. (2012). Prevalência e fatores associados à percepção negativa da saúde em pessoas idosas no Brasil. Rev Bras Epidemiol, 15(1), 49-62.

Siqueira, T., Brasil, M. R., Martins, F. Â. S., Martins, J. C. L., Weber, V. M. R., Oliveira, V. M. de, \& Silva, L. A. da. (2020). Avaliação da composição corporal e capacidade aeróbica de idosos praticantes de caminhada. Research, Society and Development, 9(6), 1-12. https://doi.org/10.33448/rsd-v9i6.3382

Soares, S. M., Dias, E., Paula, R. De, Naegle, M. A., Barbosa, P. A., Fabiano, J., Santos, G., \& Silva, L. B. (2016). Consumo de álcool e qualidade de vida em idosos na saúde da família. Revista de Enfermagem Do Centro Oeste Mineiro, 6(3), 2362-2376. https://doi.org/10.19175/recom.v6i3.1184

Sociedade Brasileira de Geriatria e Gerontologia. (2008). Quedas em Idosos: Prevenção. Associação Médica Brasileira e Conselho Federal de Medicina Quedas, 1-10.

Souza-Júnior, P. R. B. de, Freitas, M. P. S. de, Antonaci, G. de A., \& Szwarcwald, C. L. (2015). Desenho da amostra da Pesquisa Nacional de Saúde 2013. Epidemiol. Serv. Saude, 24(2), 207-216. https://doi.org/10.5123/S1679-49742015000200003

Virtuoso, J. S., Tribess, S., de Paulo, T. R. S., Martins, C. A., \& Romo-Perez, V. (2012). Atividade física como indicador preditivo para incapacidade funcional em pessoas idosas. Revista Latino-Americana de Enfermagem, 20(2), 259-265. https://doi.org/10.1590/S0104-11692012000200007

Wang, B., Wu, Y., Zhang, T., Han, J., Yu, L., \& Sun, W. (2019). Effect of physical activity on independent living ability among community-dwelling elderly 
Research, Society and Development, v. 10, n. 4, e39410414144, 2021

(CC BY 4.0) | ISSN 2525-3409 | DOI: http://dx.doi.org/10.33448/rsd-v10i4.14144

in urban areas of Liaoning Province in China: A population-based study. BMJ Open, 9(10), 1-8. https://doi.org/10.1136/bmjopen-2018-023543

Zanesco, C., Bordin, D., Santos, C. B. dos, \& Fadel, C. B. (2018). Dificuldade funcional em idosos brasileiros: Um estudo com base na pesquisa nacional de saúde (PNS - 2013). Cien Saude Colet. 